\title{
Whole Blood and Red Blood Cell Manganese Reflected Signal Intensities of T1-Weighted Magnetic Resonance Images better than Plasma Manganese in Liver Cirrhotics
}

\author{
Younghee $\mathrm{CHOI}^{1}$, Ji Kang Park ${ }^{2}$, Neung Hwa Park ${ }^{3}$, Jung Woo Shin ${ }^{3}$, Cheol-In Yoo ${ }^{1}$, \\ Choong Ryeol LeE ${ }^{1}$, Hun LeE ${ }^{1}$, Hyo Kyung KIM${ }^{4}$, Sung-Ryul KIM ${ }^{5}$, Tae-hum Jung ${ }^{6}$, \\ Jungsun PARK ${ }^{7}$, Chung Sik YoON ${ }^{8}$ and Yangho KIM ${ }^{1}$
}

\begin{abstract}
${ }^{1}$ Department of Occupational and Environmental Medicine, ${ }^{2}$ Department of Radiology, ${ }^{3}$ Department of Internal Medicine, ${ }^{4}$ Department of Neurology, ${ }^{5}$ Department of Clinical Pathology, ${ }^{6}$ Department of Family Medicine, Ulsan University Hospital, ${ }^{7}$ Korea Occupational Safety and Health Agency and ${ }^{8}$ Department of Occupational Health, Catholic University of Daegu, South Korea
\end{abstract}

\begin{abstract}
Whole Blood and Red Blood Cell Manganese Reflected Signal Intensities of T1Weighted Magnetic Resonance Images better than Plasma Manganese in Liver Cirrhotics: Younghee $\mathrm{C}_{\mathrm{HO}}$, et al. Department of Occupational and Environmental Medicine, Ulsan University Hospital, South Korea-We examined whole blood (MnB), red blood cell (MnRBC), plasma (MnP) and urinary Mn (MnU) concentrations in 22 liver cirrhotics and 10 healthy controls to evaluate Mn concentration in which a fraction of biological samples best reflects pallidal signal intensities (pallidal index; PI) on T1-weighted magnetic resonance images (MRI) in liver cirrhotics. Increased signal intensity in the globus pallidus was observed in $18(81.8 \%)$ of the 22 patients with liver cirrhosis. In a transplanted patient, increased pallidal signals also resolved as his liver function tests normalized after liver transplantation. There were significant correlations between $\mathrm{MnB} / \mathrm{MnRBC}$ and $\mathrm{PI}$ ( $\rho=0.529, \rho=0.573$, respectively) in liver cirrhotics, although no significant correlation was observed between $\mathrm{MnP} / \mathrm{MnU}$ and $\mathrm{PI}$. According to a multiple linear regression, $\mathrm{MnB}$ and $\mathrm{MnRBC}$ reflected the signal intensities of T1-weighted MRI better than MnP or MnU. (J Occup Health 2005; 47: 68-73)
\end{abstract}

Key words: Manganese, Signal intensity, Magnetic resonance, Liver cirrhosis, Pallidal index

Received June 24, 2004; Accepted Nov 15, 2004

Correspondence to: $Y$. Kim, Department of Occupational and Environmental Medicine, Ulsan University Hospital, \#290-3 Cheonha-Dong, Dong-Ku, Ulsan, 682-060, South Korea (e-mail:yanghokm@nuri.net)
Bilateral symmetrical increase in signal intensities, mainly confined to the globus pallidus (GP) and midbrain, can be observed on T1-weighted brain magnetic resonance images (MRI), but with no alteration on the T2-weighted image because of the paramagnetic quality of manganese $(\mathrm{Mn})$. The characteristic high signal intensities mainly confined to the GP have been observed in T1-weighted MRI in experimental Mn poisoning of nonhuman primates ${ }^{1)}$ and also in a patient with $\mathrm{Mn}$ neurointoxication ${ }^{2}$. Furthermore, the characteristic high signals are also frequently observed in asymptomatic workers exposed to $\mathrm{Mn}^{3}$. A similar MRI pattern has been observed in patients receiving total parenteral nutrition, because of excessive $\mathrm{Mn}$ intake ${ }^{4,5)}$. Chronic liver failure is also associated with increased signal intensities in the GP using T1-weighted MRI ${ }^{6,7)}$. The correlation between the MRI abnormalities in the GP and circulating Mn concentrations of cirrhotic patients suggests that the high signals are due to the paramagnetic $\mathrm{Mn}^{8-10)}$.

Nevertheless, Mn concentration in which partition of biological samples reflects the signal intensities of T1weighted MRI better is not known. We compared the concentrations of whole blood $\mathrm{Mn}(\mathrm{MnB})$, red blood cell $\mathrm{Mn}(\mathrm{MnRBC})$, plasma $\mathrm{Mn}(\mathrm{MnP})$ or urinary $\mathrm{Mn}(\mathrm{MnU})$ and pallidal index (PI) of T1-weighted MRI in cirrhotics and healthy controls to evaluate Mn concentration in which fraction of biological samples best reflects pallidal signal intensities in cirrhotics.

\section{Subjects and Methods}

\section{Subjects}

Male patients with clinically confirmed cirrhosis were either selected from hospital inpatients or from patients 
attending an ambulatory hepatology care clinic. The diagnosis of hepatitis B-induced liver cirrhosis was established using patient questionnaires combined with clinical, laboratory, endoscopic and ultrasonographic findings. Thirty-one patients with hepatitis B-induced liver cirrhosis gave their written consent and were recruited to the study. Nine patients were excluded because they had received transfusion due to previous gastrointestinal bleeding. The 10 male controls were selected from healthy persons visiting a health promotion center in our hospital for a comprehensive health check up including brain MRI. They agreed to participate in this study, and they were examined for blood and urine Mn concentration. These subjects had no history of liver disease or exposure to $\mathrm{Mn}$. One cirrhotic received the liver transplantation, and he was followed up after the transplantation.

\section{Questionnaires, Laboratory testing, and Analysis of Mn concentration}

Samples of heparinized venous blood and urine were collected. The heparinized venous blood was centrifugated at 3,000 rpm for $15 \mathrm{~min}$ to obtain RBC and plasma. RBC was obtained from liver cirrhotics only. A questionnaire was administered, and the questionnaire recorded basic demographic information as well as information about smoking history, alcohol consumption, medications, and recent medical history. Each subject was asked about his or her previous disease history in detail. The laboratory tests, aimed at detecting liver dysfunction or anemia, comprised of liver function tests as well as hematologic investigations such as blood counts, hemoglobin, hematocrit, and liver function tests. The severity of liver disease was assessed using the Child/ Pugh scoring system ${ }^{11)}$, which includes the variables of ascites, encephalopathy, serum albumin, serum bilirubin, and prothrombin time. Each variable is scored from 1 to 3 points for increasing abnormality and the total score equated with a Child's class (from A to C), $\mathrm{C}$ being the most severe.

Blood and urinary Mn concentrations were determined by flameless graphite furnace atomic absorption spectrophotometry (AAS) (Spectra AA880-GTA 100, Varian, Australia) ${ }^{12,13)}$. Mn concentrations were determined by the standard addition method. Aliquots $(0.1 \mathrm{ml})$ of blood were diluted 20 times with $0.1 \% \mathrm{v} / \mathrm{v}$ Triton X-100, and $15 \mu l$ samples were injected into the graphite furnace. Characteristic concentrations of $\mathrm{Mn}$ were $0.07 \mathrm{ng} / \mathrm{m} l$.

\section{Neuroimaging studies}

MRI examinations were performed using a 1.5 Tesla system (Signa Horizon LX; GE Medical Systems; Milwaukee, WI, USA). The spin echo (SE) technique was applied with two acquisitions in the T1 weighted sequence and one acquisition in the T2 weighted sequence. T1 weighted images were acquired in the axial and sagittal planes by using the following parameters: $466 / 14$ (repetition time $\left(\mathrm{T}_{\mathrm{R}}\right) /$ echo time $\left(\mathrm{T}_{\mathrm{E}}\right) \mathrm{m} \mathrm{sec}$ ] two excitations, a $22 \mathrm{~cm}$ field-of-view, a $256 \times 160$ matrix, and slice thickness of $7 \mathrm{~mm}$. T1 weighted sagittal images were also obtained using $\mathrm{T}_{\mathrm{R}} / \mathrm{T}_{\mathrm{E}}=466 / 14 \mathrm{~ms}$ with the remaining parameters as listed above. Axial T2-weighted $\left(\mathrm{T}_{\mathrm{R}} / \mathrm{T}_{\mathrm{E}}=4,000 / 100 \mathrm{~ms}\right)$ images were also obtained. The signal intensity of the GP relative to that recorded from frontal white matter was subjectively evaluated by a radiologist without prior knowledge of the disease status of the T1-weighted images. For quantitative evaluation of signal intensities, the region of interest (ROI) was placed in the region of highest intensities in the GP with visual assessment and also in the subcortical frontal white matter. We then calculated the PI, which is defined as the ratio of the signal intensity in the GP to the subcortical frontal white matter in axial T1-weighted MRI planes multiplied by $100^{9)}$.

\section{Statistical analyses}

Mean values of various continuous variables between the patients and the controls were compared using the Mann-Whitney U-test. Mean values of various continuous variables among three of the Child-Pugh class and the control group were compared using the KruskalWallis test. If the Kruskal-Wallis test showed statistical significance, the difference between each subgroup was tested by the Mann-Whitney U-test. Correlations between the $\mathrm{MnB}, \mathrm{MnRBC}, \mathrm{MnP}$, or $\mathrm{MnU}$ and the PI were evaluated using Spearman correlation coefficient (rho, $\rho)$. Analyses of multiple regression modeling were also performed to verify any association between the MnB, $\mathrm{MnRBC}$, or MnP level (explanatory variables) and the PI value (response variable) whilst controlling the influence of other explanatory variables in liver cirrhotics.

\section{Results}

The general, clinical and biochemical characteristics of the subjects are shown in Table 1. The mean age of cirrhotic patients was $48.4 \mathrm{yr}$ and all were male. All the patients had viral hepatitis B-induced liver cirrhosis and $18.2 \%$ had a previous history of hepatic encephalopathy. The 10 subjects used as controls had a mean age of 48.3 $\mathrm{yr}$ and were free of any exposure to $\mathrm{Mn}$, neurological disease, liver disease, or anemia. Patients with cirrhosis had a mean $\mathrm{MnB}$ of $2.34 \pm 1.11 \mu \mathrm{g} / \mathrm{d} l$, which was statistically different from that of the control group. The $\mathrm{MnP}$ and $\mathrm{MnU}$ in the cirrhotic patients was not statistically different from the control group. The mean PI of the cirrhotics was statistically significantly different from that of controls. Increased T1-weighted MRI signals in the GP were observed in $18(81.8 \%)$ of 22 patients 
Table 1. Demographic, clinical and laboratory features of the studied male subjects

\begin{tabular}{|c|c|c|c|c|}
\hline & \multicolumn{2}{|c|}{ Case $(n=22)$} & \multicolumn{2}{|c|}{ Control $(n=10)$} \\
\hline Age (yr) & \multicolumn{2}{|c|}{$48.4 \pm 6.43$} & \multicolumn{2}{|c|}{$48.3 \pm 6.0$} \\
\hline Alcohol drinking (premorbid) & \multicolumn{2}{|c|}{$16(72.7 \%)$} & \multicolumn{2}{|c|}{$6(60.0 \%)$} \\
\hline Hepatic encephalopathy & \multicolumn{2}{|c|}{$4(18.2 \%)$} & \multicolumn{2}{|c|}{$0 \quad(0.0 \%)$} \\
\hline High signal intensity on MRI & \multicolumn{2}{|c|}{$18 \quad(81.8 \%)$} & 0 & $(0.0 \%)$ \\
\hline Pallidal index & $122.62 \pm 14.96 * *$ & $(92.55-141.85)$ & $106.23 \pm 7.94$ & $(93.30-116.00)$ \\
\hline $\operatorname{MnB}(\mu \mathrm{g} / \mathrm{d} l)$ & $2.34 \pm 1.11^{*}$ & $(0.76-5.10)$ & $1.44 \pm 0.49$ & $(0.88-2.22)$ \\
\hline $\operatorname{MnP}(\mu \mathrm{g} / \mathrm{d} l)$ & $0.19 \pm 0.05$ & $(0.10-0.30)$ & $0.21 \pm 0.06$ & $(0.16-0.32)$ \\
\hline $\operatorname{MnRBC}(\mu \mathrm{g} / \mathrm{d} l)$ & $3.74 \pm 1.88$ & $(1.75-8.67)$ & & \\
\hline $\mathrm{MnU}(\mu \mathrm{g} / \mathrm{L})$ & $0.60 \pm 0.41$ & $(0.08-1.95)$ & $0.78 \pm 0.28$ & $(0.45-1.16)$ \\
\hline Hemoglobin $(\mathrm{g} / \mathrm{d} l)$ & $11.8 \pm 2.0^{* *}$ & $(7.4-16.0)$ & $15.8 \pm 1.2$ & $(14.6-18.8)$ \\
\hline Total bilirubin $(\mathrm{mg} / \mathrm{d} l)$ & $2.67 \pm 2.28$ & $(0.7-8.7)$ & $1.30 \pm 0.37$ & $(0.8-2.0)$ \\
\hline Direct bilirubin $(\mathrm{mg} / \mathrm{d} l)$ & $1.40 \pm 1.39 *$ & $(0.2-4.6)$ & $0.24 \pm 0.07$ & $(0.2-0.4)$ \\
\hline AST (IU/L) & $78.0 \pm 52.9 * *$ & $(27-264)$ & $31.4 \pm 10.6$ & $(19-54)$ \\
\hline ALT (IU/L) & $96.2 \pm 134.9$ & $(26-587)$ & $46.6 \pm 29.0$ & $(17-103)$ \\
\hline Child-Pugh score & $8.5 \pm 2.9$ & $(6-14)$ & & \\
\hline
\end{tabular}

Statistical significance test was done by Mann-Whitney U-test. NOTE, Data are Mean \pm standard deviation (range), *: $p<0.05,{ }^{*}: p<0.01$ vs control, MnB: whole blood manganese (Mn) concentration, MnP: plasma Mn concentration, MnRBC: RBC Mn concentration, MnU: urinary Mn concentration

Table 2. Pallidal index (PI) and blood manganese (Mn) concentrations by Child-Pugh classification

\begin{tabular}{lcccc}
\hline & Controls $(\mathrm{n}=10)$ & & Child-Pugh classification & \\
& & $\mathrm{A}(\mathrm{n}=7)$ & $\mathrm{B}(\mathrm{n}=9)$ & $\mathrm{C}(\mathrm{n}=6)$ \\
\hline $\mathrm{PI}$ & $106.23 \pm 7.94 * *$ & $110.60 \pm 13.77$ & $128.48 \pm 13.56$ s, \#\# & $127.87 \pm 11.02$ s, \#\# \\
$\mathrm{MnB}$ & $1.44 \pm 0.49$ & $1.88 \pm 0.60$ & $2.84 \pm 1.27$ & $2.14 \pm 1.16$ \\
$\mathrm{MnP}$ & $0.21 \pm 0.06$ & $0.17 \pm 0.04$ & $0.20 \pm 0.06$ & $0.18 \pm 0.06$ \\
$\mathrm{MnRBC}$ & & $3.11 \pm 1.31$ & $4.17 \pm 1.90$ & $3.83 \pm 2.48$ \\
$\mathrm{MnU}$ & $0.78 \pm 0.28$ & $0.50 \pm 0.18$ & $0.73 \pm 0.58$ & $0.54 \pm 0.26$ \\
\hline
\end{tabular}

Statistical significance test was done by Kruskal-Wallis test $(* *: p<0.01)$. If Kruskal-Wallis test show statistical significance, the difference between each subgroup was tested Mann-Whitney U-test ( $\$$ : $p<0.05$ vs class A, \#\#: $p<0.01$ vs controls). Data are Mean \pm standard deviation (range). $\mathrm{MnB}:$ whole blood Mn concentration ( $\mu \mathrm{g} / \mathrm{d} l) \mathrm{MnP}$ : plasma Mn concentration $(\mu \mathrm{g} / \mathrm{d} l)$ MnRBC $:$ RBC Mn concentration $(\mu \mathrm{g} / \mathrm{d} l) \mathrm{MnU}:$ urinary Mn concentration $(\mu \mathrm{g} / \mathrm{L})$

with liver cirrhosis. No increases in pallidal signal were observed in controls. In a transplanted patient, increased pallidal signals also resolved as his liver function tests normalized after liver transplantation (Fig. 1). However, $\mathrm{MnB}$ after transplantation $(1.28 \mu \mathrm{g} / \mathrm{d} l)$ as well as before transplantation $(1.15 \mu \mathrm{g} / \mathrm{d} l)$ were lower than mean MnB of the controls. There was a significant correlation between MnB and PI $(\rho=0.529, p<0.01 ; n=22)$ in liver cirrhotics. There was also a significant correlation between MnRBC and PI ( $p=0.573, \mathrm{P}<0.01 ; \mathrm{n}=22)$ in liver cirrhotics, but correlations between MnP and PI $(\rho=0.265$, $\rho>0.05 ; \mathrm{n}=22)$ and between MnU and PI $(\rho=-0.175$, $\rho>0.05 ; n=22)$ were not statistically significant. Mean
PI was significantly different among four groups including three groups of liver cirrhotics (Child-Pugh class A, B, C) and a control group. Especially, PI in Child/Pugh class B and C was significantly different from PI in Child/Pugh class A as well as the control group. Mean PI of Child-Pugh class A, B, and C in liver cirrhotics was $110.60,128.48$, and 127.87 , respectively, whereas mean PI in the control group was 106.23. Nevertheless, mean $\mathrm{MnB}$ was not different statistically among the four groups.

According to a multiple linear regression analysis, $\mathrm{MnB}$ increased PI after controlling for total bilirubin and age in liver cirrhotics $(p=0.022)$ (Table 3 , model 1$)$. MnRBC 


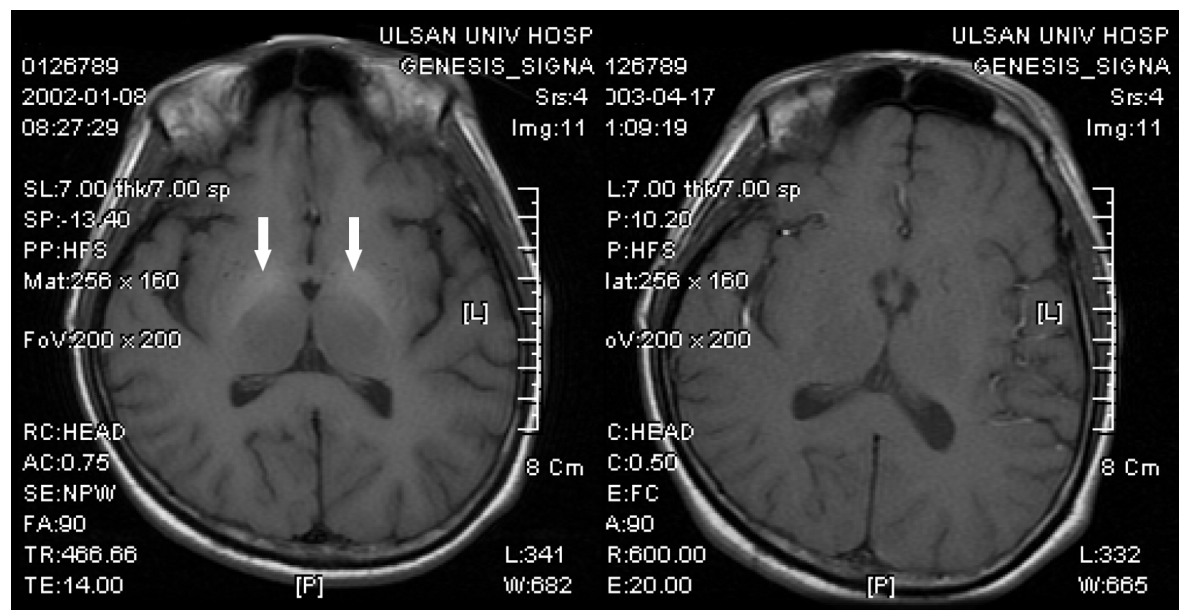

Fig. 1. (A) Axial $\mathrm{T} 1$-weighted image $(\mathrm{TR} / \mathrm{TE}=466 / 14 \mathrm{~ms})$ shows bilateral increased signal intensities at globus pallidus (white arrows) before liver transplantation. (B) The increased signal intensities in axial T1-weighted image resolved at globus pallidus after the liver transplantation.

Table 3. Results of multiple regression analysis modeling pallidal index (PI) in liver cirrhotics

\begin{tabular}{lrrrr}
\hline & Coefficient $(95 \%$ CI $)$ & $\beta$ & $p$ value & $\mathrm{R}^{2}$ \\
\hline Model 1 (n=22) & $6.246(1.027-11.465)$ & 0.462 & 0.022 & \\
MnB & $1.921(-0.617-4.459)$ & 0.292 & 0.129 & 0.405 \\
Total bilirubin & $-0.622(-1.5118-0.266)$ & -0.268 & 0.158 & \\
Age & & & 0.030 & 0.385 \\
\hline Model 2 (n=22) & $3.625(0.383-6.867)$ & 0.456 & 0.030 & 0.221 \\
MnRBC & $1.595(-1.051-4.240)$ & 0.243 & 0.096 & \\
Total bilirubin & $-0.767(-1.682-0.149)$ & -0.330 & 0.051 & \\
Age & & & 0.060 & 0.343 \\
\hline Model 3 (n=22) & $110.198(-5.315-225.710)$ & 0.385 & 0.108 & \\
MnP & $2.133(-0.517-4.783)$ & -0.278 & 0.164 & \\
Total bilirubin & $-0.646(-1.580-0.289)$ & & & \\
Age & & & & \\
\hline
\end{tabular}

$\beta$ : adjusted correlation coefficient, MnB: whole blood manganese (Mn) concentration $(\mu \mathrm{g} / \mathrm{d} l)$, MnRBC: RBC Mn concentration $(\mu \mathrm{g} / \mathrm{d} l), \mathrm{MnP}$ : plasma Mn concentration $(\mu \mathrm{g} / \mathrm{d} l)$

also increased PI after controlling for total bilirubin and age $(p=0.030)$ (Table 3, model 2), although, MnP or MnU failed to increase PI after controlling for total bilirubin and age (Table 3, model 3). Data on MnU was not presented.

\section{Discussion}

After exposure to $\mathrm{Mn}$, characteristic increased signal intensities can be observed mainly confined to the GP using T1-weighted MRI. Similar patterns of increased T1-weighted signal intensities can be associated with lipid $^{14)}$, hemoglobin breakdown products ${ }^{15)}$, melanoma $^{16)}$, neurofibromatosis ${ }^{17)}$, and calcification ${ }^{18,19}$. But iron deposits cause a more marked shortening of T2-relaxation time compared to T1-relaxation time, which results in a low signal intensity in the $\mathrm{T} 2$-weighted image, allowing clear distinction of iron and Mn deposits. Melanoma and neurofibromatosis can also be differentiated from $\mathrm{Mn}$ deposits from knowledge of the site and symmetry of the signals. Furthermore, calcification-induced signals can be ruled out by normal CT findings ${ }^{20)}$. Hence, only Mn is known to cause an abnormal increase in T1-weighted MRI signals in the same distribution and pattern as 
observed in patients with chronic liver diseases.

In our hepatitis B-induced cirrhotic patients, we noted abnormal increased T1 signals in the GP (81.8\%). In one patient, increased pallidal signals also resolved as his liver function tests normalized after liver transplantation. The present finding was compatible with the other study ${ }^{21)}$. All these findings also support that Mn causes an increase in T1-weighted MRI signals.

Previous studies ${ }^{6-8,22)}$ have shown that subjective MR imaging scores correlated with blood Mn concentrations. The present study confirmed and extended previous reports showing a correlation between increased pallidal signals and blood Mn concentrations. Here we report a correlation between blood Mn concentration and PI in T1-weighted MRI. Furthermore, MnB or MnRBC reflected the signal intensities of T1-weighted MRI better than $\mathrm{MnP}$ or MnU. The present findings are in accordance with other studies that concluded that while MnP does not provide the best picture of Mn status, MnB reflects $\mathrm{Mn}$ stores in tissue more accurately ${ }^{23-25)}$. Most of the Mn content of whole blood is in the cellular components, with the erythrocytes containing $65 \%$, leukocytes and platelets $25 \%$, and plasma $5 \%$ in subjects with normal liver function ${ }^{26)}$. It is likely that erythrocytes represent a Mn compartment more akin to target tissues than serum, and hence provide a more sensitive indicator of the $\mathrm{Mn}$ body burden. Whole blood is easier to obtain than RBC practically. Hence, $\mathrm{MnB}$ is a useful proxy indicator of target organ dose of $\mathrm{Mn}$ in the field of occupational health.

In the present study, PI in Child/Pugh class B and C was significantly different from PI in Child/Pugh class A as well as the control group. The present finding shows a significant relationship between PI and the severity of the liver disease assessed using the Child/Pugh scoring system $^{11)}$. This finding is in agreement with other studies $^{21,27,28)}$.

The present study has its limitations. First, we did not analyzed MnRBC in the controls. Second, the present study had small number of study samples, especially the controls.

In summary, $\mathrm{MnB}$ or MnRBC reflected signal intensities of T1-weighted MRI better than MnP or MnU.

Acknowledgments: This study was supported by a grant of the Korea Health 21 R\&D Project, Ministry of Health \& Welfare, Republic of Korea (01-PJ1-PG3-21900-0007).

\section{References}

1) MC Newland, TL Ceckler, JH Kordower and B Weiss: Visualizing manganese in the primate basal ganglia with magnetic resonance imaging. Exp Neurol 106, 251-258 (1989)

2) K Nelson, J Golnick, T Korn and C Angle: Manganese encephalopathy: utility of early magnetic resonance imaging. Br J Ind Med 50, 510-513 (1993)

3) Y Kim, KS Kim, JS Yang, IJ Park, E Kim, Y Jin, KR
Kwon, KH Chang, JW Kim, SH Park, HS Lin, HK Cheon, YC Shin, J Park and Y Moon: Increase in signal intensities on $\mathrm{T} 1$-weighted magnetic resonance images in asymptomatic manganese-exposed workers. Neurotoxicology 20, 901-908 (1999)

4) SA Mirowitz, TJ Westrich and JD Hirsch: Hyperintense basal ganglia on T1-weighted MR images in patients receiving parenteral nutrition. Radiology 181, 117-120 (1991)

5) A Ejima, T Imamur, S Nakamura, H Saito, K Matsumoto and S Momono: Manganese intoxication during total parental nutrition. Lancet 339, 426 (1992)

6) RF Butterworth, L Spahr, S Fontaine and GP Layrargues: Manganese toxicity, dopaminergic dysfunction and hepatic encephalopathy. Metab Brain Dis 10, 259-267 (1995).

7) RA Hauser, TA Zesiewicz, AS Rosemurgy, C Martinez and CW Olanow: Manganese intoxication and chronic liver failure. Ann Neurol 36, 871-875 (1994)

8) H Saito and A Ejima: Liver dysfunction and probable manganese accumulation in the brainstem and basal ganglia. J Neurol Neurosurg Psychiatry 58, 760-761 (1995)

9) D Krieger, S Krieger, O Jansen, P Gass, L Theilmann and H Lichtnecker: Manganese and chronic hepatic encephalopathy. Lancet 346, 270-274 (1995)

10) H Maeda, M Sato, A Yoshikawa, M Kimura, T Sonomura, M Terada and K Kishi: Brain MR imaging in patients with hepatic cirrhosis: relationship between high intensity signal in basal ganglia on T1-weighted images and elemental concentrations in brain. Neuroradiology 39, 546-550 (1997)

11) RNH Pugh, IM Murray-Lyon, JL Dawson, MC Pietroni and R Williams: Transection of the esophagus for bleeding esophageal varices. Br J Surg 60, 646-649 (1973)

12) S Baldwin, M Deaker and W Maher: Low volume microwave digestion of marine biological tissues for the measurement of trace elements. Analyst 119, 17011704 (1994)

13) B Francois, G Oliver, A Josiane and P Francis: Determination of manganese in biological materials by electrothermal atomic absorption spectrometry, a review. Clin Chem 34, 227-234 (1988)

14) WR Markesberry, WD Ehmann, T Hossain and M Alauddin: Brain manganese concentrations in human and Alzheimer's disease. Neurotoxicology 5, 49-59 (1984)

15) JM Gomori, RI Grossman, HI Goldberg, RZ Zimmerman and LT Bilaniuk: Intracranial hematomas: imaging by high-field MR. Radiology 157, 87-93 (1985)

16) JM Gomori, RI Grossman, JA Shields, JJ Augsburger, PM Joseph and D DeSimeone: Choroidal melanomas: correlation of NMR spectroscopy and MR imaging. Radiology 158, 443-445 (1986)

17) SA Mirowitz, K Sartor and M Gado: High intensity basal ganglia lesion on T1-weighted MR images in neurofibromatosis. Am J Neuroradiol 10, 1159-1163 (1989) 
18) LA Dell, MS Brown, WW Orrison, CG Eckel and NA Matwiyoff: Physiologic intracranial calcification with hyperintensity on MR imaging: case report and experimental model. Am J Neuroradiol 9, 1145-1148 (1988)

19) RM Henkelman, MF Watts and W Kucharczyk: High signal intensity in MR images of calcified brain tissue. Radiology 179, 199-206 (1991)

20) J Ahn, C-I Yoo, CR Lee, JH Lee, H Lee, JK Park, T Sakai, CS Yoon and Y Kim: Calcification mimicking manganese-induced increased signal intensities in T1weighted MR images in a patient taking herbal medicine: case report. Neurotoxicology 24, 835-838 (2003)

21) A Pujol, J Pujol, F Graus, A Rimola, J Peri, JM Mercader, JC Garcia-Pagan, J Bosch, J Rodes and E Tolosa: Hyperintense globus pallidus on T1-weighted MRI in cirrhotic patients is associated with severity of liver failure. Neurology 43, 65-69 (1993)

22) L Spahr, RF Butterworth, S Fontaine, L Bui, G Therrien, PC Milette, LH Lebrun, J Zayed, A Leblanc and G Pomier-Layrargues: Increased blood manganese in cirrhotic patients: relationship to pallidal magnetic resonance signal hyperintensity and neurological symptoms. Hepatology 24, 1116-1120 (1996)

23) CL Keen, MS Clegg, B Lonnerdal and LS Hurley:
Whole-blood manganese as an indicator of body manganese. N Engl J Med 308, 1230 (1983)

24) MS Clegg, B Lönnerdal, LS Hurley and CL Keen: Analysis of whole blood manganese by flameless atomic absorption spectrometry and its use as an indicator of manganese status in animals. Anal Biochem 157, 12-18 (1986)

25) NH Park, JK Park, Y Choi, C-I Yoo, CR Lee, H Lee, HK Kim, S-R Kim, TH Jung, J Park, CS Yoon and Y Kim: Whole blood manganese correlates with high signal intensities on T1-weighted MRI in patients with liver cirrhosis. Neurotoxicology 24, 909-915 (2003)

26) DB Milne, RL Sims and NVC Ralston: Manganese content of the cellular components of blood. Clin Chem 36, 450-452 (1990)

27) J Kulisevsky, J Pujol, J Balanzo, C Junque, J Deus, A Capdevilla and C Villanueva: Pallidal hyperintensity on magnetic resonance imaging in cirrhotic patients: clinical correlations. Hepatology 16, 1382-1388 (1992)

28) SD Taylor-Robinson, A Oatridge, JV Hajnal, AK Burroughs, N McIntyre and NM deSouza: MR imaging of the basal ganglia in chronic liver disease: correlation of T1-weighted and magnetisation transfer contrast measurements with liver dysfunction and neuropsychiatric status. Metab Brain Dis 10, 175-188 (1995) 